\title{
A GENERALIZATION OF A THEOREM OF WHITE
}

\author{
VICTOR BATYREV AND JOHANNES HOFSCHEIER
}

\begin{abstract}
An $m$-dimensional simplex $\Delta$ in $\mathbb{R}^{m}$ is called empty lattice simplex if $\Delta \cap \mathbb{Z}^{m}$ is exactly the set of vertices of $\Delta$. A theorem of White states that if $m=3$ then, up to an affine unimodular transformation of the lattice $\mathbb{Z}^{m}$, any empty lattice simplex $\Delta \subset \mathbb{R}^{3}$ is isomorphic to a tetrahedron whose vertices have third coordinate 0 or 1 . In this paper, we prove a generalization of this theorem for some special empty lattice simplices of arbitrary odd dimension $m=2 d-1$ which was conjectured by Sebő and Borisov. Our result implies a classification of all $2 d$-dimensional isolated Gorenstein cyclic quotient singularities with minimal log-discrepancy $\geq d$.
\end{abstract}

\section{INTRODUCTION}

We work with polytopes $\Delta$ in the $m$-dimensional real space $\mathbb{R}^{m}$ containing the standard lattice $\mathbb{Z}^{m}$. By $e_{1}, \ldots, e_{m}$ we denote the standard basis of $\mathbb{Z}^{m}$. A $k$-dimensional simplex $\Delta$ is a convex hull of affinely independent vectors $v_{1}, \ldots, v_{k+1}$ of $\mathbb{R}^{m}$ in which case $v_{1}, \ldots, v_{k+1}$ are the vertices of $\Delta$. We call $\Delta$ a lattice simplex if its vertices $v_{1}, \ldots, v_{k+1}$ are contained in $\mathbb{Z}^{m}$. Similarly a polytope $\Delta$ in $\mathbb{R}^{m}$ is called a lattice polytope if its vertices are in $\mathbb{Z}^{m}$. A lattice simplex $\Delta$ is called empty if $\Delta \cap \mathbb{Z}^{m}$ is the set of its vertices. In [Whi64] White posed the problem to investigate general properties of empty lattice simplices and, if possible, classify them. By "classification" one means a classification up to a natural notion of isomorphism, namely up to affine linear isomorphisms respecting the lattice $\mathbb{Z}^{m}$. Many mathematicians have already worked directly or indirectly on this question [Whi64, Mor85, MMM88, Seb99, HZ00, Bor08, BBBK11, IVS18, IVnS19a, IVnS19b, CS20]. In [Whi64] White gave a full classification of 3-dimensional empty lattice simplices.

Theorem 1.1. (WhITE) Let $\Delta \subset \mathbb{R}^{3}$ be a 3-dimensional lattice simplex, i.e., a lattice tetrahedron. Then the following statements are equivalent:

(1) $\Delta$ is empty;

(2) $\Delta$ is affine unimodularly isomorphic to a lattice simplex $\operatorname{conv}\left(v_{1}, v_{2}, v_{3}, v_{4}\right) \subset \mathbb{R}^{3}$ such that the third coordinate of $v_{1}, v_{2}$ is 0 , the third coordinate of $v_{3}, v_{4}$ is 1 , and the edges $\Delta_{1}=\operatorname{conv}\left(v_{1}, v_{2}\right), \Delta_{2}=\operatorname{conv}\left(v_{3}, v_{4}\right)$ are empty.

The aim of the present paper is to generalize this theorem to an arbitrary odd dimension $2 d-1(d \geq 2)$. For this generalization we need some additional definitions.

First, we need the notion of unimodular lattice simplices.

2000 Mathematics Subject Classification. Primary 52B20; Secondary 14B05, 11B68.

Key words and phrases. Empty lattice simplices, Ehrhart theory, $h^{*}$-polynomial, Bernoulli functions, quotient singularity. 


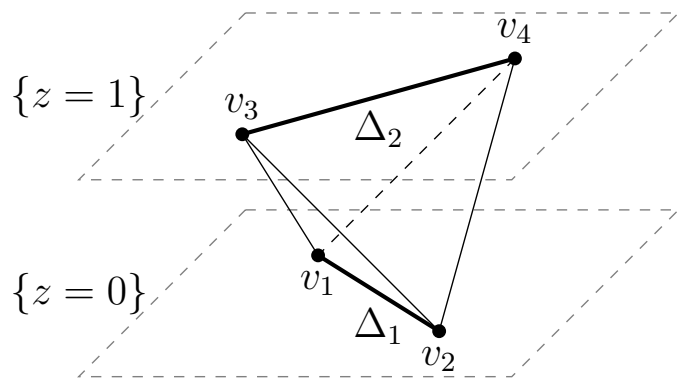

FiguRE 1. Illustration to the theorem of White (see Theorem 1.1).

Definition 1.2. (Unimodular lattice Simplex) A $k$-dimensional lattice simplex

$$
\Delta=\operatorname{conv}\left(v_{1}, \ldots, v_{k+1}\right) \subset \mathbb{R}^{m}
$$

is called a unimodular lattice simplex if one of the following two equivalent conditions hold:

(1) There is an affine unimodular isomorphism $\alpha: \mathbb{Z}^{m} \rightarrow \mathbb{Z}^{m}$ such that

$$
\alpha(\Delta)=\operatorname{conv}\left(0, e_{1}, \ldots, e_{k}\right) ;
$$

(2) $v_{1}-v_{k+1}, v_{2}-v_{k+1}, \ldots, v_{k}-v_{k+1}$ is part of a lattice basis for $\mathbb{Z}^{m}$.

Remark 1.3. Note that if a 3 -dimensional lattice simplex $\Delta \subset \mathbb{R}^{3}$ is empty, then all its codimension 1 faces are unimodular lattice simplices (i.e., unimodular lattice triangles).

Second, we use the notion of a Cayley polytope (see for instance [BN07] or [BN08]).

Definition 1.4. (CAyley Polytope) Let $\Delta_{1}, \ldots, \Delta_{r} \subset \mathbb{R}^{m}$ be $r$ lattice polytopes. Consider the cone

$$
\sigma:=\left\{\left(\lambda_{1}, \ldots, \lambda_{r}, \sum \lambda_{i} \Delta_{i}\right) \subset \mathbb{R}^{m+r} \mid \lambda_{i} \geq 0\right\} .
$$

The intersection of $\sigma$ with the hyperplane

$$
H_{r}:=\left\{\left(x_{1}, \ldots, x_{m+r}\right) \in \mathbb{R}^{m+r} \mid \sum_{i=1}^{r} x_{i}=1\right\}
$$

is called the Cayley polytope of $\Delta_{1}, \ldots, \Delta_{r}$ and will be denoted by $\Delta_{1} * \ldots * \Delta_{r}$. It is straightforward to show that $\Delta_{1} * \ldots * \Delta_{r}$ is the convex hull of the polytopes $e_{1} \times$ $\Delta_{1}, \ldots, e_{r} \times \Delta_{r}$ in $\mathbb{R}^{m+r}$. In particular, $\Delta_{1} * \ldots * \Delta_{r}$ is a lattice polytope.

Remark 1.5. Using the notion of Cayley polytope, we can reformulate the theorem of White (Theorem 1.1) in the following equivalent form: $\Delta \subset \mathbb{R}^{3}$ is an empty lattice tetrahedron if and only if $\Delta$ is isomorphic to a Cayley polytope of two empty lattice segments $\Delta_{1}:=\operatorname{conv}\left(v_{1}, v_{2}\right) \subset \mathbb{R}^{2}$ and $\Delta_{2}:=\operatorname{conv}\left(v_{3}, v_{4}\right) \subset \mathbb{R}^{2}$, i.e., $\Delta \cong \Delta_{1} * \Delta_{2}$.

Definition 1.6. (LATTICE SIMPLEX $\Delta\left(a_{1}, \ldots, a_{d-1} ; n\right)$ ) Let $a_{1}, \ldots, a_{d-1}, n$ be positive integers with $\operatorname{gcd}\left(a_{i}, n\right)=1(1 \leq i \leq d-1)$. Consider the following 1-dimensional empty lattice simplices:

$$
\Delta_{i}:=\operatorname{conv}\left(0, e_{i}\right) \subset \mathbb{R}^{d}(i=1, \ldots, d-1), \quad \Delta_{d}:=\operatorname{conv}\left(0,\left(a_{1}, \ldots, a_{d-1}, n\right)\right) \subset \mathbb{R}^{d} .
$$


Define

$$
\Delta\left(a_{1}, \ldots, a_{d-1} ; n\right):=\Delta_{1} * \cdots * \Delta_{d} \subset \mathbb{R}^{2 d-1} .
$$

Remark 1.7. It straightforwardly follows that the theorem of White (Theorem 1.1) is equivalent to the following statement: $\Delta \subset \mathbb{R}^{3}$ is an empty lattice tetrahedron if and only if $\Delta$ is isomorphic to a Cayley polytope of two empty lattice segments $\Delta_{1}:=\operatorname{conv}\left(0, e_{1}\right) \subset \mathbb{R}^{2}$ and $\Delta_{2}:=\operatorname{conv}\left(0, a_{1} e_{1}+n e_{2}\right) \subset \mathbb{R}^{2}$ for some integers $a_{1}, n$ with $\operatorname{gcd}\left(a_{1}, n\right)=1$, i.e., $\Delta \cong \Delta\left(a_{1} ; n\right)$.

We also need the notion of $h^{*}$-polynomial of a lattice polytope (see [Sta80]).

Definition 1.8. ( $h^{*}$-Polynomial of a lattice polytope) Let $\Delta \subset \mathbb{R}^{d}$ be a $d$ dimensional lattice polytope. Denote by $\left|k \Delta \cap \mathbb{Z}^{d}\right|$ the number of lattice points contained in the $k$ th dilate of $\Delta$. The Ehrhart series of $\Delta$ is a rational function

$$
1+\sum_{k \geq 1}\left|k \Delta \cap \mathbb{Z}^{d}\right| t^{k}=\frac{h_{0}^{*}+h_{1}^{*} t+\ldots+h_{d}^{*} t^{d}}{(1-t)^{d+1}}
$$

where $h_{\Delta}^{*}(t):=\sum h_{i}^{*} t^{i}$ is called the $h^{*}$-polynomial of $\Delta$. The $h^{*}$-polynomial of $\Delta$ has the following properties:

(1) all coefficients $h_{k}^{*}(0 \leq k \leq d)$ of $h_{\Delta}^{*}(t)$ are non-negative integers;

(2) $h_{0}^{*}=1, h_{1}^{*}=\left|\Delta \cap \mathbb{Z}^{d}\right|-d-1, h_{d}^{*}=\left|\operatorname{Int}(\Delta) \cap \mathbb{Z}^{d}\right|$, i.e., $h_{d}^{*}$ coincides with the number of lattice points contained in the interior of $\Delta$;

(3) $h_{\Delta}^{*}(1)=\sum_{i=0}^{d} h_{i}^{*}=\operatorname{Vol}_{d}(\Delta)$, where $\operatorname{Vol}_{d}(\Delta)$ is the lattice normalized $d$-dimensional volume of $\Delta$.

Remark 1.9. It directly follows from the above properties of $h^{*}$-polynomials that a 3 dimensional lattice polytope $\Delta \subset \mathbb{R}^{3}$ is an empty lattice tetrahedron (as in the theorem of White) if and only if its $h^{*}$-polynomial has the form

$$
h_{\Delta}^{*}(t)=1+(n-1) t^{2},
$$

where $n=\operatorname{Vol}_{3}(\Delta)$ is the lattice normalized volume of $\Delta$.

Finally, we call a set of 1-dimensional simplices $\operatorname{conv}\left(v_{i}, w_{i}\right) \subset \mathbb{R}^{d}(i=1, \ldots, r)$ linearly independent if the vectors $v_{i}-w_{i} \in \mathbb{R}^{d}$ for $i=1, \ldots, r$ are linearly independent. The codimension 1 faces of a polytope $\Delta$ are called its facets.

Now we are ready to formulate our generalization of the theorem of White:

Theorem 1.10. (GENERALIZED THEOREM OF White) Let $\Delta \subset \mathbb{R}^{2 d-1}$ be a $(2 d-1)$ dimensional lattice simplex. Then the following conditions on $\Delta$ are equivalent:

(1) $h_{\Delta}^{*}(t)=1+(n-1) t^{d}$, where $n=\operatorname{Vol}_{2 d-1}(\Delta)$ is the lattice normalized volume of $\Delta$.

(2) there exist positive integers $a_{1}, \ldots, a_{d-1}, n$ with $\operatorname{gcd}\left(a_{i}, n\right)=1(1 \leq i \leq d-1)$ such that

$$
\Delta \cong \Delta\left(a_{1}, \ldots, a_{d-1} ; n\right) .
$$

(3) $\Delta \cong \Delta_{1} * \ldots * \Delta_{d}$ for some linearly independent 1 -dimensional empty lattice simplices $\Delta_{i} \subset \mathbb{R}^{d}(i=1, \ldots, d)$, and all facets of $\Delta$ are $(2 d-2)$-dimensional unimodular lattice simplices. 
Remark 1.11. In the next section, we show that our generalization of the theorem of White was expected by Sebö in [Seb99, Conjecture 4.1].

We have organized the paper as follows. In Section 2, we prove two equivalent formulations of condition (1) from Theorem 1.10 which are used in the proof of Theorem 1.10. In Section 3, we prove a number theoretic result about Bernoulli functions on which the proof of Theorem 1.10 relies. In Section 4, we give the proof of Theorem 1.10. Section 5 concludes the paper with an application of our results to the classification of some isolated cyclic quotient singularities.

\section{ACKNOWLEDGEMENT}

The authors would like to thank Christian Haase, Benjamin Nill, and Francisco Santos for extremely useful feedback on preliminary versions of the manuscript. The second author is supported by a Nottingham Research Fellowship from the University of Nottingham.

\section{LATTICE SIMPLiCES AND THEIR $h^{*}$-POLYNOMIALS}

In this section, we give another equivalent formulation of the first condition in Theorem 1.10 using some properties of $h^{*}$-polynomials of lattice simplices (see [BR15]).

Proposition 2.1. ( $h^{*}$-POLYNOMIAL OF A LATTICE SIMPlex) Let $\Delta$ be an m-dimensional lattice simplex of $\mathbb{R}^{m}$ with vertices $v_{1}, v_{2}, \ldots, v_{m+1}$. We set $w_{j}:=\left(v_{j}, 1\right) \in \mathbb{R}^{m+1}=\mathbb{R}^{m} \times \mathbb{R}$ $(1 \leq j \leq m+1)$. Then the $k$-th coefficient $h_{k}^{*}$ in the $h^{*}$-polynomial $h_{\Delta}^{*}(t)=\sum_{i=0}^{m} h_{i}^{*} t^{i}$ equals the number of lattice points in the parallelepiped

$$
\operatorname{par}(\Delta):=\left\{\lambda_{1} w_{1}+\lambda_{2} w_{2}+\ldots+\lambda_{m+1} w_{m+1} \mid 0 \leq \lambda_{1}, \lambda_{2}, \ldots, \lambda_{m+1}<1\right\} \subset \mathbb{R}^{m+1}
$$

with last coordinate $k$.

We denote the interior of an $m$-dimensional polytope $\Delta$ in $\mathbb{R}^{m}$ by $\operatorname{Int}(\Delta)$. We prove two other equivalent characterizations of condition (1) from Theorem 1.10.

Theorem 2.2. Let $\Delta \subset \mathbb{R}^{2 d-1}$ be a $(2 d-1)$-dimensional lattice simplex with vertices $v_{1}, \ldots, v_{2 d}$. Then the following conditions on $\Delta$ are equivalent:

(1) $h_{\Delta}^{*}=1+(n-1) t^{d}$, where $n=\operatorname{Vol}_{2 d-1}(\Delta)$ is the lattice normalized volume of $\Delta$;

(2) for all $k=1, \ldots, d-1, \operatorname{Int}(k \Delta) \cap \mathbb{Z}^{2 d-1}=\emptyset$ and all facets of $\Delta$ are $(2 d-2)$ dimensional unimodular lattice simplices;

(3) for all $k=1, \ldots, d-1, k \Delta \cap \mathbb{Z}^{2 d-1} \subset \mathbb{Z} v_{1}+\ldots+\mathbb{Z} v_{2 d}$.

In the proof of Theorem 2.2, we use the fractional part $\{x\}$ of a real number $x$ which is defined as $\{x\}=x-\lfloor x\rfloor$ where $\lfloor x\rfloor$ denotes the largest integer that is less than or equal to $x$. The function $x \mapsto\lfloor x\rfloor$ is called the floor function.

Proof. $(3) \Rightarrow(2)$. Suppose condition (3) holds. We claim that

$\operatorname{par}\left(\operatorname{conv}\left(\left(v_{i}, 1\right): i \neq j\right)\right) \cap \mathbb{Z}^{2 d}=\left\{\sum_{i \neq j} \lambda_{i}\left(v_{i}, 1\right): 0 \leq \lambda_{i}<1\right\} \cap \mathbb{Z}^{2 d}=\{0\} \quad$ for $j=1, \ldots, 2 d$. 
Assume towards a contradiction that $0 \neq(w, k):=\sum_{i \neq j} \lambda_{i}\left(v_{i}, 1\right) \in \mathbb{Z}^{2 d}$ for some $0 \leq \lambda_{i}<1$ where $k=\sum_{i \neq j} \lambda_{i} \in \mathbb{Z}_{>0}$. Since, by condition (3), $w \notin l \Delta$ for $l=1, \ldots, d-1$, it follows that $k \geq d$. Consider the lattice vector $0 \neq w^{\prime}:=\sum_{i \neq j}\left\{1-\lambda_{i}\right\} v_{i} \in \mathbb{Z}^{2 d-1}$. We have $\sum_{i \neq j}\left\{1-\lambda_{i}\right\} \leq 2 d-1-\sum_{i \neq j} \lambda_{i}<d$, i.e., $w^{\prime} \in k^{\prime} \Delta$ for some $k^{\prime} \leq d-1$. Contradiction.

By [Gru07, Corollary 21.2], there exists $w_{2 d} \in \mathbb{Z}^{2 d}$ such that $\left(v_{1}, 1\right), \ldots,\left(v_{2 d-1}, 1\right), w_{2 d}$ is a basis of $\mathbb{Z}^{2 d}$. Notice we may assume that the last coordinate of $w_{2 d}$ is 1 . Thus for $j \in\{1, \ldots, 2 d\}$ the facet $\Delta_{j} \prec \Delta$ that does not contain $v_{j}$ is a $(2 d-2)$-dimensional unimodular lattice simplex. Since for all $k=1, \ldots, d-1$

$$
\operatorname{Int}(k \Delta) \cap \mathbb{Z}^{2 d-1}=\left\{\sum_{i} \lambda_{i} v_{i}: \sum_{i} \lambda_{i}=k, \lambda_{i}>0 \text { for all } i=1, \ldots, 2 d\right\},
$$

it follows by condition (3) that $\operatorname{Int}(k \Delta) \cap \mathbb{Z}^{2 d-1}=\emptyset$.

$(2) \Rightarrow(1)$. Suppose condition (2) holds. Consider $\operatorname{par}(\Delta)=\left\{\sum_{i} \lambda_{i}\left(v_{i}, 1\right) \mid 0 \leq \lambda_{i}<1\right\}$. By Proposition 2.1, the kth coefficient of $h_{\Delta}^{*}(t)$ equals to the number of lattice points contained in $\operatorname{par}(\Delta) \cap\left\{x_{2 d}=k\right\}$. Let $w:=\sum_{i} \lambda_{i}\left(v_{i}, 1\right) \in \operatorname{par}(\Delta) \cap\left\{x_{2 d}=k\right\} \cap \mathbb{Z}^{2 d}$, i.e., $0 \leq \lambda_{i}<1$ with $\sum_{i} \lambda_{i}=k \in \mathbb{Z}$. Since all facets of $\Delta$ are $(2 d-2)$-dimensional unimodular lattice simplices, it follows that $\lambda_{i}=0$ for all $i$, or $\lambda_{i}>0$ for all $i$. Let $k>0$, then all $\lambda_{i}>0$ for all $i$, i.e., $w=\sum_{i} \lambda_{i} v_{i} \in \operatorname{Int}(k \Delta) \cap \mathbb{Z}^{2 d-1}$. Hence $k \geq d$. We claim that $k \leq d$ as well. Indeed, assume towards a contradiction that $k>d$. Then $0 \neq w^{\prime}:=\sum_{i}\left\{1-\lambda_{i}\right\} v_{i} \in \operatorname{par}(\Delta) \cap \mathbb{Z}^{2 d-1}$ with $\sum_{i}\left\{1-\lambda_{i}\right\} \leq 2 d-\sum_{i} \lambda_{i}<d$. The latter contradicts the assumption (notice $\left\{1-\lambda_{i}\right\}>0$ ). Hence, we have seen that all coefficients of $h_{\Delta}^{*}(t)$ are 0 except for the $0 t h$ and the dth, i.e., $h_{\Delta}^{*}(t)=1+(n-1) t^{d}$ for the integer $n=h_{\Delta}^{*}(1)=\operatorname{Vol}_{2 d-1}(\Delta)$.

$(1) \Rightarrow(3)$. Suppose condition (1) holds. Then

$$
\operatorname{par}(\Delta) \cap\left\{x_{2 d}=k\right\} \cap \mathbb{Z}^{2 d}=\emptyset \quad \text { for all } k=1, \ldots, d-1 .
$$

Let $w:=\sum_{i} \lambda_{i} v_{i} \in k \Delta \cap \mathbb{Z}^{2 d-1}$ for $0 \leq \lambda_{i}$ with $\sum_{i} \lambda_{i}=k \in\{1, \ldots, d-1\}$. Then $w^{\prime}:=\sum_{i}\left\{\lambda_{i}\right\} v_{i} \in \mathbb{Z}^{2 d-1}$ with $0 \leq\left\{\lambda_{i}\right\}<1$ and $0 \leq l:=\sum_{i}\left\{\lambda_{i}\right\} \leq \sum_{i} \lambda_{i}=k \leq d-1$, i.e., $\sum_{i}\left\{\lambda_{i}\right\}\left(v_{i}, 1\right) \in \operatorname{par}(\Delta) \cap\left\{x_{2 d}=l\right\} \cap \mathbb{Z}^{2 d}$ for an integer $0 \leq l \leq d-1$. The case $l>0$ is not possible by our assumption. Thus, $l=0$. This implies $\left\{\lambda_{i}\right\}=0$ for all $i$, i.e., $\lambda_{i} \in \mathbb{Z}$, and $w=\sum_{i} \lambda_{i} v_{i} \in \mathbb{Z} v_{1}+\ldots+\mathbb{Z} v_{2 d}$.

\section{The Method of Morrison And Stevens}

The proof of the generalized theorem of White (Theorem 1.10) is based on a method of Morrison and Stevens that uses some number theoretic properties of the 1-st periodic Bernoulli function whose Fourier series expansion is given by

$$
\bar{B}_{1}(x):=-\sum_{|n| \geq 1} \frac{e^{2 \pi i n x}}{2 \pi i n}=-\frac{1}{\pi} \sum_{n \geq 1} \frac{\sin 2 \pi n x}{n} .
$$


By standard Fourier analysis, this series converges pointwise to the sawtooth function $\{x\}-\frac{1}{2}$, where $\{y\}:=y-\lfloor y\rfloor$ denotes the fractional part of $y \in \mathbb{R}$. Thus, we get

$$
\bar{B}_{1}(x)= \begin{cases}\{x\}-\frac{1}{2} & , \text { if } x \notin \mathbb{Z} \\ 0 & , \text { if } x \in \mathbb{Z}\end{cases}
$$

Remark 3.1. In general, one sets $\bar{B}_{0}(x):=1$ and defines the $l$-th periodic Bernoulli function $\bar{B}_{l}(x)(l \geq 1)$ as

$$
\bar{B}_{l}(x):=-\frac{l !}{(2 \pi i)^{l}} \sum_{|n| \geq 1} \frac{e^{2 \pi i n x}}{n^{l}} .
$$

Morrison and Stevens used the following statement [MS84, Section 1, Corollary 1.3]:

Theorem 3.2. ${ }^{1}$ Let $d, n$ be positive integers $(n \geq 2)$ and let $a_{1}, \ldots, a_{d}$ be integers such that $\operatorname{gcd}\left(a_{i}, n\right)=1(1 \leq i \leq d)$. Suppose for all $t \in \mathbb{Z}$ one has

$$
\sum_{i=1}^{d} \bar{B}_{1}\left(\frac{t a_{i}}{n}\right)=0 .
$$

Then the integer $d$ is even and after reordering the integers $a_{i}$ we get $a_{i}+a_{i+1} \equiv 0(\bmod n)$ for all $i=1,3,5, \ldots, d-1$.

We give a complete proof of Theorem 3.2 for arbitrary $d$.

Remark 3.3. We remark that Theorem 3.2 is a special case of a conjecture of Borisov [Bor97, Conjecture 2].

Notice that the case $n=2$ in Theorem 3.2 is straightforward. Hence in the following $n \geq 3$. We consider the finite abelian group $G_{n}:=(\mathbb{Z} / n \mathbb{Z})^{*}$, i.e., the group of units in the finite ring $\mathbb{Z} / n \mathbb{Z}$. Since $n \geq 3$, we have $\left|G_{n}\right|=\varphi(n) \geq 2$ and $g \neq-g$ for all $g \in G_{n}$. Denote by $\mathbb{C}\left[G_{n}\right]$ the group algebra of $G_{n}$ over $\mathbb{C}$, i.e., $\mathbb{C}\left[G_{n}\right]=\left\{\sum_{g \in G_{n}} a_{g} \sigma_{g}: a_{g} \in \mathbb{C}\right\}$, where the elements $\sigma_{g}$ for $g \in G_{n}$ form a canonical $\mathbb{C}$-basis of $\mathbb{C}\left[G_{n}\right]$. Since $G_{n}$ naturally acts on $\mathbb{Z} / n \mathbb{Z}$ by multiplication, and $\mathbb{Z} / n \mathbb{Z}$ is canonically isomorphic to the subgroup $\frac{1}{n} \mathbb{Z} / \mathbb{Z} \subset \mathbb{R} / \mathbb{Z}$, the value $\bar{B}_{1}(g x)$ is well-defined for any $g \in G_{n}$ and for any $x \in \frac{1}{n} \mathbb{Z} / \mathbb{Z}$.

For any $x \in \frac{1}{n} \mathbb{Z} / \mathbb{Z}$, we consider the Stickelberger element

$$
S(x):=\sum_{g \in G_{n}} \bar{B}_{1}(g x) \sigma_{g} \in \mathbb{C}\left[G_{n}\right],
$$

and denote by $U$ the $\mathbb{C}$-vector subspace of $\mathbb{C}\left[G_{n}\right]$ generated by all Stickelberger elements $S(x)$, i.e., $U:=\operatorname{span}\left\{S(x): x \in \frac{1}{n} \mathbb{Z} / \mathbb{Z}\right\}$.

Let $\left\{\sigma_{g}^{*}: g \in G_{n}\right\}$ be the basis of the dual vector space $\widehat{\mathbb{C}\left[G_{n}\right]}$ which is dual to the canonical basis $\left\{\sigma_{g}: g \in G_{n}\right\}$ of $\mathbb{C}\left[G_{n}\right]$. We denote by

$$
\langle\cdot, \cdot\rangle: \mathbb{C}\left[G_{n}\right] \times \widehat{\mathbb{C}\left[G_{n}\right]} \rightarrow \mathbb{C},\langle v, f\rangle:=f(v)
$$

\footnotetext{
${ }^{1}$ Morrison and Stevens only gave a proof for $d=4$. A similar result has been shown by Reid in [Rei87, Appendix to §5]. A generalization of Theorem 3.2 using Reid's method was found by Celaya [Cel18].
} 
the natural dual pairing. The key idea is to show that a basis of the orthogonal complement

$$
U^{\perp}=\left\{f \in \widehat{\mathbb{C}\left[G_{n}\right]}: f(u)=0 \text { for all } u \in U\right\}
$$

of the subspace $U \subset \mathbb{C}\left[G_{n}\right]$ is given by the $\varphi(n) / 2$ elements $u_{g}^{*}:=\sigma_{g}^{*}+\sigma_{-g}^{*}$ for $g \in G_{n}$ :

Lemma 3.4. $\left\{u_{g}^{*}:=\sigma_{g}^{*}+\sigma_{-g}^{*}: g \in G_{n}\right\}$ is a basis of $U^{\perp}$. In particular $\operatorname{dim}_{\mathbb{C}}\left(U^{\perp}\right)=$ $\operatorname{dim}_{\mathbb{C}}(U)=\varphi(n) / 2$.

We postpone the proof of Lemma 3.4 until the end of this section and now complete the proof of Theorem 3.2.

Proof of Theorem 3.2. We consider the element

$$
u^{*}:=\sigma_{\overline{a_{1}}}^{*}+\sigma_{\overline{a_{2}}}^{*}+\ldots+\sigma_{\overline{a_{d}}}^{*} \in \widehat{\mathbb{C}\left[G_{n}\right]},
$$

where $\overline{a_{1}}, \ldots, \overline{a_{d}}$ are the elements of $G_{n}=(\mathbb{Z} / n \mathbb{Z})^{*}$ corresponding to the integers $a_{1}, \ldots, a_{d}$. From the assumptions in Theorem 3.2, it follows that for all integers $t \in \mathbb{Z}$ we have

$$
\begin{aligned}
\left\langle S\left(\frac{t}{n}\right), u^{*}\right\rangle & =\left\langle\sum_{g \in G_{n}} \bar{B}_{1}\left(\frac{t g}{n}\right) \sigma_{g}, \sigma_{\overline{a_{1}}}^{*}+\sigma_{\overline{a_{2}}}^{*}+\ldots+\sigma_{\overline{a_{d}}}^{*}\right\rangle \\
& =\bar{B}_{1}\left(\frac{t a_{1}}{n}\right)+\bar{B}_{1}\left(\frac{t a_{2}}{n}\right)+\ldots+\bar{B}_{1}\left(\frac{t a_{d}}{n}\right)=0 .
\end{aligned}
$$

Thus $u^{*} \in U^{\perp}$. On the other hand, we can write $u^{*}=\sum_{g \in G_{n}} k_{g} \sigma_{g}^{*}$ for some nonnegative integral coefficients $k_{g}\left(g \in G_{n}\right)$, where $k_{g}$ is the nonnegative multiplicity of the basis vector $\sigma_{g}^{*}$ in the sum $\sum_{i=1}^{d} \sigma_{\overline{a_{i}}}^{*}$. By Lemma 3.4, we can write $u^{*}$ as a unique linear combination of sums $\sigma_{g}^{*}+\sigma_{-g}^{*}$ over all $\varphi(n) / 2$ pairs $\{g,-g\}$, i.e., there exists a set of $\varphi(n) / 2$ coefficients $\lambda_{\{g,-g\}}$ corresponding to pairs $\{g,-g\}$ such that

$$
\begin{gathered}
u^{*}=\sum_{g \in G_{n}} k_{g} \sigma_{g}^{*}=\sum_{\{g,-g\} \subset G_{n}} \lambda_{\{g,-g\}}\left(\sigma_{g}^{*}+\sigma_{-g}^{*}\right), \\
d=\sum_{g \in G_{n}} k_{g}=2 \sum_{\{g,-g\} \subset G_{n}} \lambda_{\{g,-g\}} .
\end{gathered}
$$

Hence $k_{g}=k_{-g}=\lambda_{\{g,-g\}}$ for all $g \in G_{n}$, and $d \in 2 \mathbb{Z}$. Thus one can reorder the integers $\left\{a_{i}\right\}_{i=1}^{d}$ into pairs $\left\{a_{i}, a_{i+1}\right\}$ for $i=1,3, \ldots, d-1$ such that $a_{i}+a_{i+1} \equiv 0(\bmod n)$.

It remains to show Lemma 3.4.

Proof of Lemma 3.4. Consider the regular representation $\rho: G \rightarrow \mathrm{GL}\left(\mathbb{C}\left[G_{n}\right]\right)$ of $G_{n}$, i.e., for all $g \in G_{n}$

$$
\rho_{g}: \mathbb{C}\left[G_{n}\right] \rightarrow \mathbb{C}\left[G_{n}\right], \sigma_{h} \mapsto \sigma_{g h}
$$

Since $G_{n}$ is a finite abelian group, the $\mathbb{C}$-space $\mathbb{C}\left[G_{n}\right]$ splits into a direct sum of $\varphi(n)=$ $\left|G_{n}\right|$ 1-dimensional invariant subspaces $W_{i} \subset \mathbb{C}\left[G_{n}\right](i=1, \ldots, \varphi(n))$ corresponding to pairwise different characters $\chi_{1}, \ldots, \chi_{\varphi(n)}$. By simultaneous diagonalization of commuting endomorphisms $\rho_{g}\left(g \in G_{n}\right)$, we obtain another $\mathbb{C}$-basis $e_{1}, \ldots, e_{\varphi(n)}$ of $\mathbb{C}\left[G_{n}\right]$ consisting of orthogonal idempotents $e_{i} \in \mathbb{C}\left[G_{n}\right]$ such that for all $i \in\{1, \ldots, \varphi(n)\}$ one has $e_{i}^{2}=e_{i}$, 
$\rho_{g}\left(e_{i}\right)=\chi_{i}(g) e_{i}$ (for all $\left.g \in G_{n}\right), e_{i} e_{j}=0$ for $i \neq j$, and $1=\sum_{i=1}^{\varphi(n)} e_{i}$. Let $\mathcal{X}(n):=$ $\left\{\chi_{1}, \ldots, \chi_{\varphi(n)}\right\}$ be the group of characters of $G_{n}$. Then $\mathcal{X}(n) \cong G_{n}$, but this isomorphism is not canonical. By well-known properties of characters (see for instance [Isa06, Theorem 2.12]), we obtain the following relation between the two bases $e_{1}, \ldots, e_{\varphi(n)}$ and $\left\{\sigma_{g}\right\}_{g \in G_{n}}$ :

$$
\sigma_{g}=\sum_{i=1}^{\varphi(n)} \chi_{i}(g) e_{i} \quad \text { for all } g \in G_{n}
$$

and

$$
e_{i}=\frac{1}{\varphi(n)} \sum_{g \in G_{n}} \chi_{i}\left(g^{-1}\right) \sigma_{g} \quad \text { for all } i \in\{1, \ldots, \varphi(n)\} .
$$

One has $\mathcal{X}(n)=\mathcal{X}^{+}(n) \sqcup \mathcal{X}^{-}(n)$, where $\mathcal{X}^{+}(n):=\{\chi \in \mathcal{X}(n): \chi(-1)=1\}$ is the subgroup of even characters and $\mathcal{X}^{-}(n):=\{\chi \in \mathcal{X}(n): \chi(-1)=-1\}$ is the set of odd characters. Obviously, one has $\left|\mathcal{X}^{+}(n)\right|=\left|\mathcal{X}^{-}(n)\right|=\varphi(n) / 2$.

For any $\chi \in \mathcal{X}(n)$ there exists a minimal positive integer $C_{\chi}$ dividing $n$ such that the character $\chi: G_{n}=(\mathbb{Z} / n \mathbb{Z})^{*} \rightarrow \mathbb{C}^{*}$ factors through the natural homomorphism $(\mathbb{Z} / n \mathbb{Z})^{*} \rightarrow$ $\left(\mathbb{Z} / C_{\chi} \mathbb{Z}\right)^{*}$. The integer $C_{\chi}$ is called the conductor of $\chi$. We denote by the same letter $\chi$ the lift of $\chi: G_{n}=(\mathbb{Z} / n \mathbb{Z})^{*} \rightarrow \mathbb{C}^{*}$ to its corresponding Dirichlet character. This is a function $\chi: \mathbb{Z} \rightarrow \mathbb{C}$ satisfying the following conditions

(1) $\chi(a)=\chi(b)$ if $a \equiv b\left(\bmod C_{\chi}\right)$;

(2) $\chi(a b)=\chi(a) \chi(b)$ for all $a, b \in \mathbb{Z}$;

(3) $\chi(a)=0$ if $\operatorname{gcd}\left(a, C_{\chi}\right) \neq 1$;

(4) $\chi(a)=\chi(\bar{a})$ if $\operatorname{gcd}\left(a, C_{\chi}\right)=1, \bar{a}=a+C_{\chi} \mathbb{Z}$, where $\chi$ on the right hand side is considered as the unique homomorphism $\chi:\left(\mathbb{Z} / C_{\chi} \mathbb{Z}\right)^{*} \rightarrow \mathbb{C}^{*}$.

Since $C_{\chi} \mid n$, the values of the function $\chi: \mathbb{Z} \rightarrow \mathbb{C}$ on elements $g$ of $G_{n}$ are well-defined. To any character $\chi \in \mathcal{X}(n)$, one assigns a complex number

$$
B_{1, \chi}:=\sum_{k=1}^{C_{\chi}} \chi(k) \bar{B}_{1}\left(\frac{k}{C_{\chi}}\right) .
$$

In [Was97, Chapter 4], the numbers $B_{1, \chi}$ are called generalized Bernoulli numbers. We will need the following nontrivial result on the nonvanishing of $B_{1, \chi}{ }^{2}$.

Theorem 3.5. If $\chi$ is an odd Dirichlet character, then $B_{1, \chi} \neq 0$.

Theorem 3.5 is a direct consequence of the following three statements from classical number theory (see for instance [BS66, Ch. V, §2], or [Rib01, Ch. 21 and Ch. 22]):

Theorem 3.6. Let $\chi$ be a non-trivial Dirichlet character and let

$$
L(s, \chi)=\sum_{n=1}^{\infty} \frac{\chi(n)}{n^{s}}, \operatorname{Re}(s)>0
$$

\footnotetext{
${ }^{2}$ Washington writes in [Was97, p. 38]: “... Note that the theorem implies that $B_{1, \chi} \neq 0$ if $\chi$ is odd. There is no elementary proof known for this fact...".
} 
be the corresponding Dirichlet L-function. Then $L(1, \chi) \neq 0$.

Theorem 3.7. [BS66, Ch. V, §2, Theorem 3] Let $\chi$ be an odd Dirichlet character with conductor $C_{\chi}$. Denote by $\bar{\chi}$ the conjugate odd character. Then

$$
L(1, \chi)=\pi i \frac{\tau(\chi)}{C_{\chi}^{2}} \sum_{k=1}^{C_{\chi}} \bar{\chi}(k) k=\pi i \frac{\tau(\chi)}{C_{\chi}} B_{1, \bar{\chi}},
$$

where $\tau(\chi)$ denotes a Gauss sum

$$
\tau(\chi)=\sum_{a=1}^{C_{\chi}} \chi(a) e^{2 \pi i a / C_{\chi}} .
$$

Theorem 3.8. Let $\chi$ be a Dirichlet character with conductor $C_{\chi}$. Then the absolute value $|\tau(\chi)|$ of the Gauss sum $\tau(\chi)$ is $\sqrt{C_{\chi}}$. In particular, $\tau(\chi) \neq 0$.

Take an odd Dirichlet character $\chi_{i}$ induced by an odd character $\chi_{i} \in \mathcal{X}^{-}(n)$ of $G_{n}$. By Theorem 3.5, we have $B_{1, \chi_{i}} \neq 0$. We rescale the vector $e_{i} \in \mathbb{C}\left[G_{n}\right]$ by the non-zero factor $\left|G_{n}\right| B_{1, \chi_{i}}$ and obtain

$$
\begin{aligned}
u_{\chi_{i}}:=\left|G_{n}\right| B_{1, \chi_{i}} e_{i} & =B_{1, \chi_{i}} \sum_{g \in G_{n}} \chi_{i}\left(g^{-1}\right) \sigma_{g}=\sum_{g \in G_{n}} B_{1, \chi_{i}} \chi_{i}\left(g^{-1}\right) \sigma_{g} \\
& =\sum_{g \in G_{n}} \sum_{k=1}^{C_{\chi_{i}}} \underbrace{\chi_{i}(k) \chi_{i}\left(g^{-1}\right)}_{\chi_{i}\left(k g^{-1}\right)} \bar{B}_{1}\left(\frac{k}{C_{\chi_{i}}}\right) \sigma_{g} \\
k^{\prime}: & =k g^{-1} \\
= & \sum_{g \in G_{n}} \sum_{k^{\prime}=1}^{C_{\chi_{i}}} \chi_{i}\left(k^{\prime}\right) \bar{B}_{1}\left(\frac{k^{\prime} g}{C_{\chi_{i}}}\right) \sigma_{g} \\
& =\sum_{k^{\prime}=1}^{C_{\chi_{i}}} \chi_{i}\left(k^{\prime}\right) \sum_{g \in G_{n}} \bar{B}_{1}\left(\frac{k^{\prime} g}{C_{\chi_{i}}}\right) \sigma_{g}=\sum_{k^{\prime}=1}^{C_{\chi_{i}}} \chi_{i}\left(k^{\prime}\right) S\left(\frac{k^{\prime}}{C_{\chi_{i}}}\right) \in U .
\end{aligned}
$$

It follows that for all $\chi \in \mathcal{X}^{-}(n)$ the vector $u_{\chi}$ is contained in $U$. Since the $e_{i}$ are linearly independent, we obtain a linearly independent set $\left\{u_{\chi}: \chi \in \mathcal{X}^{-}(n)\right\}$. Hence $\operatorname{dim}_{\mathbb{C}}(U) \geq$ $\varphi(n) / 2$. Since $\operatorname{dim}_{\mathbb{C}}(U)+\operatorname{dim}_{\mathbb{C}}\left(U^{\perp}\right)=\operatorname{dim}_{\mathbb{C}}\left(\mathbb{C}\left[G_{n}\right]\right)=\varphi(n)$ it follows that $\operatorname{dim}_{\mathbb{C}}\left(U^{\perp}\right) \leq$ $\varphi(n) / 2$. On the other hand, the $\varphi(n) / 2$ elements $u_{g}^{*}=\sigma_{g}^{*}+\sigma_{-g}^{*}$ are contained in $U^{\perp}$, because for all $x \in \frac{1}{n} \mathbb{Z} / \mathbb{Z}$ one has

$$
\left\langle S(x), u_{g}^{*}\right\rangle=\left\langle\sum_{g \in G_{n}} \bar{B}_{1}(g x) g, \sigma_{g}^{*}+\sigma_{-g}^{*}\right\rangle=\bar{B}_{1}(g x)+\bar{B}_{1}(-g x)=0 .
$$

Moreover, the $\varphi(n) / 2$ elements $\left\{u_{g}^{*}: g \in G_{n}\right\}$ are linearly independent, since $\left\{\sigma_{g}^{*}: g \in G_{n}\right\}$ form a basis of $\widehat{\mathbb{C}\left[G_{n}\right]}$. Thus, we obtain the opposite inequality $\operatorname{dim}_{\mathbb{C}}\left(U^{\perp}\right) \geq \varphi(n) / 2$. Hence, $\operatorname{dim}_{\mathbb{C}}\left(U^{\perp}\right)=\varphi(n) / 2$ and the set $\left\{u_{g}^{*}: g \in G_{n}\right\}$ is a basis of $U^{\perp}$. Lemma 3.4 is proved. 


\section{The Proof of Theorem 1.10}

In this section we prove Theorem 1.10. We need the following statement which was implicitly used in the proof of Theorem 2.2.

Proposition 4.1. Let $\Delta=\operatorname{conv}\left(v_{1}, \ldots, v_{2 d}\right) \subset \mathbb{R}^{2 d-1}$ be a $(2 d-1)$-dimensional lattice simplex with $h_{\Delta}^{*}(t)=1+(n-1) t^{d}$, where $n=\operatorname{Vol}_{2 d-1}(\Delta)$ (see Theorem 2.2). Suppose that for some rational numbers $0 \leq \lambda_{i}<1(i \in\{1, \ldots, 2 d\})$ the linear combination $\sum_{i=1}^{2 d} \lambda_{i}\left(v_{i}, 1\right) \in \mathbb{Z}^{2 d}$ is a lattice vector. Then either $\sum_{i} \lambda_{i}=0$, or $\sum_{i} \lambda_{i}=d$. Furthermore, if $\sum_{i} \lambda_{i}=d$, then $\lambda_{i} \neq 0$ for all $i$.

Proof. By Proposition 2.1 and our assumption, if $w=\sum_{i=1}^{2 d} \lambda_{i}\left(v_{i}, 1\right) \in \mathbb{Z}^{2 d}$ is a lattice vector for some rational numbers $0 \leq \lambda_{i}<1(i \in\{1, \ldots, 2 d\})$, then either $\sum_{i} \lambda_{i}=0$ (if $w=0$ ) or $\sum_{i} \lambda_{i}=d$ (if $w \neq 0$ ).

To show the second part of the statement, assume towards a contradiction that there is a lattice vector as above $0 \neq w=\sum_{i=1}^{2 d} \lambda_{i}\left(v_{i}, 1\right) \in \mathbb{Z}^{2 d}$ but with, e.g., $\lambda_{1}=0$. Then $0 \neq \sum_{i=1}^{2 d}\left\{1-\lambda_{i}\right\}\left(v_{i}, 1\right) \in \mathbb{Z}^{2 d}$ with $0 \leq\left\{1-\lambda_{i}\right\}<1(i \in\{1, \ldots, 2 d\})$ and $\sum_{i=1}^{2 d}\left\{1-\lambda_{i}\right\} \leq$ $2 d-1-\sum_{i=1}^{2 d} \lambda_{i}=d-1$. A contradiction to our assumption on $\Delta$.

Proof of Theorem 1.10. (1) $\Rightarrow(2)$. Let $\Delta=\operatorname{conv}\left(v_{1}, \ldots, v_{2 d}\right) \subset \mathbb{R}^{2 d-1}$ be a $(2 d-1)$ dimensional simplex such that $h_{\Delta}^{*}(t)=1+(n-1) t^{d}$. By Theorem 2.2, all facets of $\Delta$ are $(2 d-2)$-dimensional unimodular lattice simplices and for all $k=1, \ldots, d-1$, $\operatorname{Int}(k \Delta) \cap \mathbb{Z}^{2 d-1}=\emptyset$. Therefore, the facet $\Gamma:=\operatorname{conv}\left(v_{1}, \ldots, v_{2 d-1}\right) \subset \Delta$ is a $(2 d-2)$ dimensional unimodular lattice simplex, and thus we can extend the set of lattice vectors $\left(v_{1}, 1\right), \ldots,\left(v_{2 d-1}, 1\right)$ to a $\mathbb{Z}$-basis

$$
\left(v_{1}, 1\right), \ldots,\left(v_{2 d-1}, 1\right),(w, l) \in \mathbb{Z}^{2 d}=\mathbb{Z}^{2 d-1} \times \mathbb{Z} .
$$

Hence the lattice vector $\left(v_{2 d}, 1\right) \in \mathbb{Z}^{2 d}$ can be written as an integral linear combination:

$$
\left(v_{2 d}, 1\right)=n_{1}\left(v_{1}, 1\right)+\cdots+n_{2 d-1}\left(v_{2 d-1}, 1\right)+n(w, l),
$$

where the last coefficient $n$ equals $\operatorname{Vol}_{2 d-1}(\Delta)$. We set

$$
\Delta^{\prime}:=\operatorname{conv}\left(\left(v_{1}, 1\right), \ldots,\left(v_{2 d}, 1\right)\right) \subset \mathbb{R}^{2 d}
$$

such that $\Delta^{\prime} \cong \Delta$. Consider the finite abelian group

$$
G\left(\Delta^{\prime}\right):=\mathbb{Z}^{2 d} /\left(\mathbb{Z}\left(v_{1}, 1\right)+\ldots+\mathbb{Z}\left(v_{2 d}, 1\right)\right),
$$

which is generated by $(w, l)$ such that $G\left(\Delta^{\prime}\right) \cong \mathbb{Z} / n \mathbb{Z}$. Therefore, we can choose the lattice vector $(w, l) \in \mathbb{Z}^{2 d} \cap \operatorname{par}(\Delta)$ as a rational linear combination

$$
(w, l)=\sum_{i=1}^{2 d} \mu_{i}\left(v_{i}, 1\right), \quad \mu_{i} \in \frac{1}{n} \mathbb{Z}, \quad 0 \leq \mu_{i}<1 .
$$

We write

$$
\mu_{i}=\frac{a_{i}}{n} \text { for some integers } 0 \leq a_{i}<n(i=1, \ldots, 2 d)
$$


By Proposition 4.1 , for all $t \in \mathbb{Z} \backslash n \mathbb{Z}$ and for all $i=1, \ldots, 2 d$, one has $\left\{t a_{i} / n\right\} \neq 0$, i.e., $\operatorname{gcd}\left(a_{i}, n\right)=1$ for all $i=1, \ldots, 2 d$. The last condition implies that for all $t \in \mathbb{Z} \backslash n \mathbb{Z}$

$$
0 \neq \sum_{i=1}^{2 d}\left\{\frac{t a_{i}}{n}\right\}\left(v_{i}, 1\right) \in \operatorname{par}(\Delta) \cap \mathbb{Z}^{2 d} .
$$

This shows that we can assume $a_{2 d}=1$. Furthermore, we obtain for all $t \in \mathbb{Z} \backslash n \mathbb{Z}$

$$
d=\sum_{i=1}^{2 d}\left\{\frac{t a_{i}}{n}\right\} \text { (by Proposition 4.1) } \Leftrightarrow 0=\sum_{i=1}^{2 d} \bar{B}_{1}\left(\frac{t a_{i}}{n}\right) .
$$

The right hand side of this equation is satisfied for all integers $t \in n \mathbb{Z}$, since $\bar{B}_{1}(k)=0$ for all integers $k \in \mathbb{Z}$. By Theorem 3.2, we can assume (after reordering the lattice vectors $\left.v_{1}, \ldots, v_{2 d-1}\right)$ that $a_{2 i-1}+a_{2 i} \equiv 0(\bmod n)$ for all $i=1, \ldots, d$ (we can leave the lattice vector $v_{2 d}$ at its place, i.e., $\left.a_{2 d-1}=n-1, a_{2 d}=1\right)$.

Let $\alpha$ be the unique unimodular linear isomorphism which maps the basis

$$
\left\{\left(v_{1}, 1\right), \ldots,\left(v_{2 d-1}, 1\right),(w, l)-\left(v_{2}, 1\right)-\left(v_{4}, 1\right)-\ldots-\left(v_{2 d-2}, 1\right)\right\} \subset \mathbb{Z}^{2 d-1} \times \mathbb{Z}
$$

of $\mathbb{Z}^{2 d}$ to the basis

$$
\left\{\left(e_{1}, 0\right),\left(e_{1}, e_{1}\right),\left(e_{2}, 0\right),\left(e_{2}, e_{2}\right), \ldots,\left(e_{d}, 0\right),\left(e_{d}, e_{d}\right)\right\} \subset \mathbb{Z}^{d} \times \mathbb{Z}^{d} .
$$

With the equations $a_{2 i-1}+a_{2 i}=n$ for all $i=1, \ldots, d$ and the linear relations

$$
\left(v_{2 d}, 1\right)=n(w, l)-\sum_{i=1}^{2 d-1} a_{i}\left(v_{i}, 1\right), \quad\left(e_{d}, e_{d}\right)=\alpha((w, l))-\sum_{i=1}^{d-1}\left(e_{i}, e_{i}\right),
$$

we obtain

$$
\begin{aligned}
\alpha\left(\left(v_{2 d}, 1\right)\right) & =n \alpha((w, l))-\sum_{i=1}^{2 d-1} a_{i} \alpha\left(\left(v_{i}, 1\right)\right) \\
& =n\left(e_{d}, e_{d}\right)+n\left(\sum_{i=1}^{d-1}\left(e_{i}, e_{i}\right)\right)-a_{2 d-1}\left(e_{d}, 0\right)-\left(\sum_{i=1}^{d-1} a_{2 i-1}\left(e_{i}, 0\right)+a_{2 i}\left(e_{i}, e_{i}\right)\right) \\
& =n\left(e_{d}, e_{d}\right)+\left(\sum_{i=1}^{d-1}\left(a_{2 i-1}\left(e_{i}, e_{i}\right)-a_{2 i-1}\left(e_{i}, 0\right)\right)\right)-(n-1)\left(e_{d}, 0\right) \\
& =\left(e_{d}, n e_{d}\right)+\sum_{i=1}^{d-1}\left(0, a_{2 i-1} e_{i}\right) \\
& =\left(e_{d}, a_{1} e_{1}+a_{3} e_{2}+a_{5} e_{3}+\cdots+a_{2 d-3} e_{d-1}+n e_{d}\right) .
\end{aligned}
$$

Hence $\Delta$ is affine unimodularly isomorphic to $\Delta_{1} * \ldots * \Delta_{d}$ for

$$
\begin{aligned}
& \Delta_{i}:=\operatorname{conv}\left(0, e_{i}\right) \subset \mathbb{R}^{d} \text { for } i=1, \ldots, d-1 \text { and } \\
& \Delta_{d}:=\operatorname{conv}\left(0,\left(a_{1}, a_{3}, a_{5}, \ldots, a_{2 d-3}, n\right)\right) \subset \mathbb{R}^{d}
\end{aligned}
$$

This proves $(1) \Rightarrow(2)$. 
$(2) \Rightarrow(3)$. Suppose that $\Delta=\Delta\left(a_{1}, \ldots, a_{d-1} ; n\right)$ for some positive integers $a_{1}, \ldots, a_{d-1}, n$ with $\operatorname{gcd}\left(a_{i}, n\right)=1$ for all $i \in\{1, \ldots, d-1\}$. Clearly, then $\Delta$ is isomorphic to a Cayley polytope $\Delta_{1} * \cdots * \Delta_{d}$ for some linearly independent 1-dimensional empty lattice simplices $\Delta_{i} \subset \mathbb{R}^{d}$. Moreover, $G\left(\Delta^{\prime}\right) \cong \mathbb{Z} / n \mathbb{Z}$, where

$$
\Delta^{\prime}:=(\Delta, 1)=\left(\Delta\left(a_{1}, \ldots, a_{d-1} ; n\right), 1\right) \subset \mathbb{R}^{2 d} .
$$

Now we can directly compute the $h^{*}$-polynomial of $\Delta$ and obtain $h_{\Delta}^{*}(t)=1+(n-1) t^{d}$. By Theorem 2.2, it follows that all facets of $\Delta$ are $(2 d-2)$-dimensional unimodular lattice simplices. This proves $(2) \Rightarrow(3)$.

(3) $\Rightarrow(1)$. If $\Delta=\Delta_{1} * \ldots * \Delta_{d}$ is a Cayley polytope, then we obtain a surjective lattice projection $\pi: \Delta \rightarrow \Sigma_{d-1}$, where $\Sigma_{d-1}$ is a $(d-1)$-dimensional unimodular lattice simplex. Since $k \Sigma_{d-1}$ has no interior lattice points for $k \leq d-1$, it follows that

$$
\operatorname{Int}(k \Delta) \cap \mathbb{Z}^{2 d-1}=\emptyset \quad \text { for all } k \in\{1, \ldots, d-1\} .
$$

By Theorem 2.2, this implies that $h_{\Delta}^{*}(t)=1+(n-1) t^{d}$, where $n=\operatorname{Vol}_{2 d-1}(\Delta)$. This proves $(3) \Rightarrow(1)$.

Theorem 1.10 together with Theorem 2.2 implies the following expanded version of a generalization of Theorem 1.1.

Corollary 4.2. (EXPANDED VERSION OF THE GENERALIZED THEOREM OF White) Let $\Delta \subset \mathbb{R}^{2 d-1}$ be a $(2 d-1)$-dimensional lattice simplex. Then the following conditions on $\Delta$ are equivalent:

(1) For all $k=1, \ldots, d-1, k \Delta \cap \mathbb{Z}^{2 d-1} \subset \mathbb{Z} v_{1}+\ldots+\mathbb{Z} v_{2 d}$.

(2) For all $k=1, \ldots, d-1$, $\operatorname{Int}(k \Delta) \cap \mathbb{Z}^{2 d-1}=\emptyset$ and all facets of $\Delta$ are $(2 d-2)$ dimensional unimodular lattice simplices.

(3) $h_{\Delta}^{*}=1+(n-1) t^{d}$, where $n=\operatorname{Vol}_{2 d-1}(\Delta)$ is the lattice normalized volume of $\Delta$.

(4) $\Delta$ is isomorphic to one of the simplices $\Delta\left(a_{1}, \ldots, a_{d-1} ; n\right)$ from Definition 1.6.

(5) $\Delta \cong \Delta_{1} * \ldots * \Delta_{d}$ for some linearly independent 1-dimensional empty lattice simplices $\Delta_{i} \subset \mathbb{R}^{d}$, and all facets of $\Delta$ are $(2 d-2)$-dimensional unimodular lattice simplices.

As an illustration we consider the case of 5 -dimensional lattice simplices (i.e., $d=3$ ).

Corollary 4.3. Let $\Delta \subset \mathbb{R}^{5}$ be a 5-dimensional empty lattice simplex with $\operatorname{Vol}_{5}(\Delta)=n$. Then the following statements are equivalent:

(1) $h_{\Delta}^{*}=1+(n-1) t^{3}$, where $n=\operatorname{Vol}_{5}(\Delta)$ is the lattice normalized volume of $\Delta$.

(2) $\Delta$ is isomorphic to one of the lattice simplices $\Delta\left(a_{1}, a_{2} ; n\right)$ from Definition 1.6.

(3) $\Delta \cong \Delta_{1} * \Delta_{2} * \Delta_{3}$ for linearly independent 1-dimensional lattice simplices $\Delta_{i} \subset \mathbb{R}^{3}$, and all lattice points contained in $2 \Delta \cap \mathbb{Z}^{5}$ are either the vertices of the simplex $2 \Delta$, or the midpoints of its edges.

Finally, we give an example which shows that the assumption about lattice points in $2 \Delta$ in Corollary 4.3(3) cannot be dropped, in other words, one cannot omit the assumption that all codimension 1 faces of $\Delta$ are unimodular lattice simplices in Theorem 1.10(3). 
Example 4.4. Let $p \neq q$ be two prime integers and consider the following linearly independent segments in $\mathbb{R}^{3}$ :

$$
\Delta_{1}:=\operatorname{conv}(0,(1,0,0)), \quad \Delta_{2}:=\operatorname{conv}(0,(1, p, 0)), \quad \Delta_{3}:=\operatorname{conv}(0,(1,0, q)) .
$$

Then the Cayley polytope $\Delta_{1} * \Delta_{2} * \Delta_{3}$ is an empty 5-dimensional lattice simplex in the affine lattice plane $\left\{x_{1}+x_{2}+x_{3}=1\right\} \subset \mathbb{R}^{6}$ and it is isomorphic to

$$
\Delta=\operatorname{conv}\left(0 \times \Delta_{1}, e_{1} \times \Delta_{2}, e_{2} \times \Delta_{3}\right) \subset \mathbb{R}^{5}=\mathbb{R}^{2} \times \mathbb{R}^{3} .
$$

Notice the doubled simplex $2 \Delta$ contains lattice points which are not an integer linear combination of the vertices of $\Delta$, namely for $k=1, \ldots, p-1$ and $l=1, \ldots, q-1$, we have

$$
(1,0,1, k, 0)=\frac{k}{p}(1,0,1, p, 0)+\frac{p-k}{p}(1,0,0,0,0)+\frac{p-k}{p}(0,0,1,0,0)+\frac{k}{p}(0,0,0,0,0)
$$

and

$$
(0,1,1,0, l)=\frac{l}{q}(0,1,1,0, q)+\frac{q-l}{q}(0,1,0,0,0)+\frac{q-l}{q}(0,0,1,0,0)+\frac{l}{q}(0,0,0,0,0) .
$$

We claim that there is an isomorphism

$$
G(\Delta):=\mathbb{Z}^{6} /\left(\mathbb{Z}\left(v_{1}, 1\right)+\ldots+\mathbb{Z}\left(v_{6}, 1\right)\right) \cong \mathbb{Z} / p \mathbb{Z} \oplus \mathbb{Z} / q \mathbb{Z} \cong \mathbb{Z} / p q \mathbb{Z},
$$

where $v_{i}$ are the vertices of $\Delta$ defined above. Indeed every element of $G(\Delta)$ has a unique representative in $\operatorname{par}(\Delta)$. By [Cas97] or [Seb90, Lemma 2], the total number of lattice points contained in $\operatorname{par}(\Delta)$ equals to the determinant of the matrix, whose rows consist of the vertices of $\Delta$ and added a column with ones. It is straightforward to show that the determinant of this matrix is $p q$. The remaining non-zero elements in $G(\Delta)$ are induced by lattice points in $3 \Delta$, namely for $k=1, \ldots, p-1$ and $l=1, \ldots, q-1$

$$
\begin{aligned}
\frac{k}{p}(1,0,1, p, 0)+\frac{p-k}{p}(1,0,0,0,0)+ & \frac{l}{q}(0,1,1,0, q)+\frac{q-l}{q}(0,1,0,0,0)+ \\
& \left\{-\frac{k q+l p}{p q}\right\}(0,0,1,0,0)+\left\{\frac{k q+l p}{p q}\right\}(0,0,0,0,0) .
\end{aligned}
$$

Thus, the $h^{*}$-polynomial of $\Delta$ equals

$$
h_{\Delta}^{*}(t)=1+(p+q-2) t^{2}+(p-1)(q-1) t^{3} .
$$

By Theorem 1.10, this implies $\Delta ¥ \Delta\left(a_{1}, a_{2} ; p q\right)$.

\section{Classification of SOME isolated CyCliC QuOtient Singularities}

Let us now explain how Theorem 1.10 gives rise to a full classification of $2 d$-dimensional isolated cyclic quotient singularities with minimal log-discrepancy $\geq d$.

An $m$-dimensional cyclic quotient singularity is an affine variety obtained as the quotient of $\mathbb{C}^{m}$ by a linear action of the cyclic group $\mu_{n}$ of $n$-th roots of unity. After diagonalizing, we can always assume that the $\mu_{n}$-actions is given by

$$
\mu_{n} \times \mathbb{C}^{m} \rightarrow \mathbb{C}^{m}, \quad\left(\zeta,\left(x_{1}, \ldots, x_{m}\right)\right) \mapsto\left(\zeta^{a_{1}} x_{1}, \ldots, \zeta^{a_{m}} x_{d}\right)
$$


for some integers $a_{i}(1 \leq i \leq m)$, where $\zeta \in \mathbb{C}$ is a primitive $n$-th root of unity. We call the rational vector $\left(\frac{a_{1}}{n}, \ldots, \frac{a_{m}}{n}\right) \in \mathbb{Q}^{m}$ the type of the quotient singularity. If $a_{i}=0$ for some $i \in\{1, \ldots, m\}$, then the quotient $X=\mathbb{C}^{m} / \mu_{n}$ is isomorphic to a product $X^{\prime} \times \mathbb{C}$, where $X^{\prime}=\mathbb{C}^{m-1} / \mu_{n}$ is a lower-dimensional quotient singularity. Hence we may assume that $a_{i} \neq 0$ for all $i$. The quotient singularity $\mathbb{C}^{m} / \mu_{n}$ of type $\left(\frac{a_{1}}{n}, \ldots, \frac{a_{m}}{n}\right)$ has an isolated singularity at the origin if and only if $\operatorname{gcd}\left(a_{i}, n\right)=1$ for all $i=1, \ldots, m$ (see [MS84, Corollary 2.2]). To a cyclic quotient singularity one associates the minimal log-discrepancy. In the cyclic case it has the following combinatorial description (see [Bor97] or [Rei83]):

Definition 5.1. Let $\mathbb{C}^{m} / \mu_{n}$ be an isolated cyclic quotient singularity of type $\left(\frac{a_{1}}{n}, \ldots, \frac{a_{m}}{n}\right)$. Then the minimal log-discrepancy is given by

$$
\min _{t \in\{1, \ldots, n-1\}} \sum_{i=1}^{m}\left\{\frac{t a_{i}}{n}\right\} .
$$

Furthermore, a quotient singularity $\mathbb{C}^{m} / \mu_{n}$ is called Gorenstein if the image of the homomorphism $\mu_{n} \rightarrow \mathrm{GL}(m ; \mathbb{C})$ induced by the linear $\mu_{n}$-action on $\mathbb{C}^{m}$ is contained in $\mathrm{SL}(m ; \mathbb{C})$. This property is easy to see from the type of the quotient singularity:

Proposition 5.2. Let $X=\mathbb{C}^{m} / \mu_{n}$ be a cyclic quotient singularity of type $\left(\frac{a_{1}}{n}, \ldots, \frac{a_{m}}{n}\right)$. Then $X$ is Gorenstein if and only if

$$
\sum_{i=1}^{m} \frac{a_{i}}{n} \in \mathbb{Z} .
$$

Now we can prove the following theorem.

Theorem 5.3. Let $\mathbb{C}^{2 d} / \mu_{n}$ be an isolated cyclic quotient singularity of type $\left(\frac{a_{1}}{n}, \ldots, \frac{a_{2 d}}{n}\right)$. Then the following two statements are equivalent:

(1) The minimal log-discrepancy of the quotient singularity is at least $d$.

(2) After reordering the integers $a_{i}$, one obtains $a_{2 i-1}+a_{2 i} \equiv 0(\bmod n)$ for all $i=$ $1, \ldots, d$. In other words, the $\mu_{n}$-action on $\mathbb{C}^{2 d}$ is determined by a diagonal matrix

$$
\operatorname{diag}\left(\zeta^{a_{1}}, \zeta^{-a_{1}}, \zeta^{a_{3}}, \zeta^{-a_{3}}, \ldots, \zeta^{a_{2 d-1}}, \zeta^{-a_{2 d-1}}\right),
$$

where $\zeta$ is a primitive $n$-th root of unity.

Proof. The statement $(2) \Rightarrow(1)$ easily follows by the general fact that $\{x\}+\{-x\}=1$ for all $x \in \mathbb{R} \backslash \mathbb{Z}$. Thus, we obtain

$$
\sum_{i=1}^{2 d}\left\{\frac{t a_{i}}{n}\right\}=d \quad \text { for all } t \in\{1, \ldots, n-1\} .
$$

Therefore, the minimal log-discrepancy of $\mathbb{C}^{2 d} / \mu_{n}$ is $d$.

$(1) \Rightarrow(2)$. Suppose now that $\left(\frac{a_{1}}{n}, \ldots, \frac{a_{2 d}}{n}\right)$ is an isolated cyclic quotient singularity with minimal $\log$-discrepancy $\geq d$, i.e., $\operatorname{gcd}\left(a_{i}, n\right)=1$ and

$$
\sum_{i=1}^{2 d}\left\{\frac{t a_{i}}{n}\right\} \geq d \quad \text { for all } t \in\{1, \ldots, n-1\} .
$$


We claim that $\sum_{i=1}^{2 d}\left\{t a_{i} / n\right\}=d$ for all $t \in\{1, \ldots, n-1\}$. Indeed, assume towards a contradiction that there exists $t \in\{1, \ldots, n-1\}$ such that $\sum_{i=1}^{2 d}\left\{t a_{i} / n\right\}>d$. Then

$$
\sum_{i=1}^{2 d}\left\{\frac{(n-t) a_{i}}{n}\right\}=\sum_{i=1}^{2 d}\left\{\frac{-t a_{i}}{n}\right\}=2 d-\sum_{i=1}^{2 d}\left\{\frac{t a_{i}}{n}\right\}<d
$$

contradicting the fact that the minimal log-discrepancy is at least $d$. Thus, we have

$$
0=\sum_{i=1}^{2 d}\left(\left\{\frac{t a_{i}}{n}\right\}-\frac{1}{2}\right)=\sum_{i=1}^{2 d} \bar{B}_{1}\left(\frac{t a_{i}}{n}\right) \quad \text { for all } t \in\{1, \ldots, n-1\} .
$$

The assertion follows from Theorem 3.2.

Remark 5.4. The singularities classified in Theorem 5.3 are automatically Gorenstein, because

$$
\sum_{i=1}^{m}\left\{\frac{t a_{i}}{n}\right\} \in\{0, d\} \quad \text { for all } t \in\{0,1, \ldots, n-1\}
$$

or because of the conditions $a_{2 i-1}+a_{2 i} \equiv 0(\bmod n)$ for all $i=1, \ldots, d$ (see Proposition 5.2).

\section{REFERENCES}

[BBBK11] M. Barile, D. Bernardi, A. Borisov, and J.-M. Kantor, On empty lattice simplices in dimension 4, Proc. Amer. Math. Soc. 139 (2011), no. 12, 4247-4253.

[BN07] V. Batyrev and B. Nill, Multiples of lattice polytopes without interior lattice points, Mosc. Math. J. 7 (2007), no. 2, 195-207, 349.

[BN08]__ Combinatorial aspects of mirror symmetry, Integer points in polyhedra - geometry, number theory, representation theory, algebra, optimization, statistics, Contemp. Math., vol. 452, Amer. Math. Soc., Providence, RI, 2008, pp. 35-66.

[Bor97] A. Borisov, Minimal discrepancies of toric singularities, Manuscripta Math. 92 (1997), no. 1, $33-45$.

[Bor08] A. Borisov, Quotient singularities, integer ratios of factorials, and the Riemann hypothesis, Int. Math. Res. Not. IMRN (2008), no. 15, Art. ID rnn052, 19.

[BR15] M. Beck and S. Robins, Computing the continuous discretely, second ed., Undergraduate Texts in Mathematics, Springer, New York, 2015, Integer-point enumeration in polyhedra, With illustrations by David Austin.

[BS66] A. I. Borevich and I. R. Shafarevich, Number theory, Translated from the Russian by Newcomb Greenleaf. Pure and Applied Mathematics, Vol. 20, Academic Press, New York-London, 1966.

[Cas97] J. W. S. Cassels, An introduction to the geometry of numbers, Classics in Mathematics, Springer-Verlag, Berlin, 1997, Corrected reprint of the 1971 edition.

[Cel18] M. Celaya, On the linear span of lattice points in a parallelepiped, Combinatorica 38 (2018), no. $6,1385-1413$.

[CS20] G. Codenotti and F. Santos, Hollow polytopes of large width, Proc. Amer. Math. Soc. 148 (2020), no. 2, 835-850.

[Gru07] P. M. Gruber, Convex and discrete geometry, Grundlehren der Mathematischen Wissenschaften [Fundamental Principles of Mathematical Sciences], vol. 336, Springer, Berlin, 2007.

[HZ00] C. Haase and G. M. Ziegler, On the maximal width of empty lattice simplices, European J. Combin. 21 (2000), no. 1, 111-119, Combinatorics of polytopes.

[Isa06] I. M. Isaacs, Character theory of finite groups, AMS Chelsea Publishing, Providence, RI, 2006, Corrected reprint of the 1976 original [Academic Press, New York]. 
[IVS18] Ò. Iglesias-Valiño and F. Santos, The complete classification of empty lattice 4-simplices, Electronic Notes in Discrete Mathematics 68 (2018), 155-160, Discrete Mathematics Days 2018.

[IVnS19a] _ Classification of empty lattice 4-simplices of width larger than two, Trans. Amer. Math. Soc. 371 (2019), no. 9, 6605-6625.

[IVnS19b] _ The complete classification of empty lattice 4-simplices, math.CO, arXiv: 1908.08933.

[MMM88] S. Mori, D. R. Morrison, and I. Morrison, On four-dimensional terminal quotient singularities, Math. Comp. 51 (1988), no. 184, 769-786.

[Mor85] S. Mori, On 3-dimensional terminal singularities, Nagoya Math. J. 98 (1985), 43-66.

[MS84] D. R. Morrison and G. Stevens, Terminal quotient singularities in dimensions three and four, Proc. Amer. Math. Soc. 90 (1984), no. 1, 15-20.

[Rei83] M. Reid, Decomposition of toric morphisms, Arithmetic and geometry, Vol. II, Progr. Math., vol. 36, Birkhäuser Boston, Boston, MA, 1983, pp. 395-418.

[Rei87] _ Young person's guide to canonical singularities, Algebraic geometry, Bowdoin, 1985 (Brunswick, Maine, 1985), Proc. Sympos. Pure Math., vol. 46, Amer. Math. Soc., Providence, RI, 1987, pp. 345-414.

[Rib01] P. Ribenboim, Classical theory of algebraic numbers, Universitext, Springer-Verlag, New York, 2001.

[Seb90] A. Sebö, Hilbert bases, caratheodory's theorem and combinatorial optimization, Proceedings of the 1st Integer Programming and Combinatorial Optimization Conference, Waterloo, Ontorio, Canada, May 28-30 1990 (Ravi Kannan and William R. Pulleyblank, eds.), University of Waterloo Press, 1990, pp. 431-455.

[Seb99] _ An introduction to empty lattice simplices, Integer programming and combinatorial optimization (Graz, 1999), Lecture Notes in Comput. Sci., vol. 1610, Springer, Berlin, 1999, pp. 400-414.

[Sta80] R. P. Stanley, Decompositions of rational convex polytopes, Ann. Discrete Math. 6 (1980), 333-342.

[Was97] L. C. Washington, Introduction to cyclotomic fields, second ed., Graduate Texts in Mathematics, vol. 83, Springer-Verlag, New York, 1997.

[Whi64] G. K. White, Lattice tetrahedra, Canadian J. Math. 16 (1964), 389-396.

Mathematisches Institut, Universität Tübingen, Auf der Morgenstelle 10, 72076 TüBinGEN, GERMANY

Email address: victor.batyrev@uni-tuebingen.de

School of Mathematical Sciences, University of Nottingham, University Park, NotTINGHAM NG7 2RD, UK

Email address: johannes.hofscheier@nottingham.ac.uk 